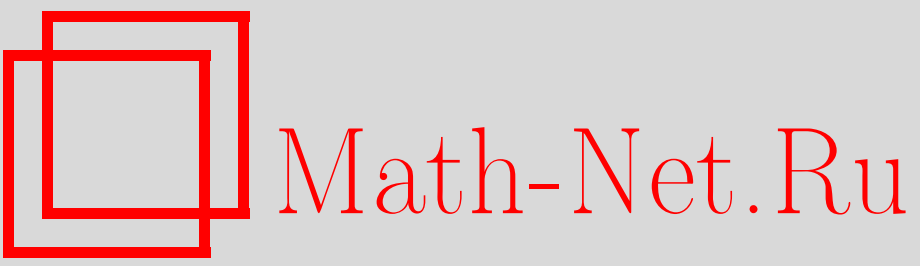

В. М. Бухштабер, Э. Г. Рис, $k$-характеры Фробениуса и $n$-кольцевые гомоморфизмы, УМH, 1997, том 52, выпуск 2, 159-160

DOI: https://doi.org/10.4213/rm830

Использование Общероссийского математического портала Math-Net.Ru подразумевает, что вы прочитали и согласны с пользовательским соглашением

http://www.mathnet.ru/rus/agreement

Параметры загрузки:

IP: 54.92.164.108

26 апреля 2023 г., 18:28:41 


\title{
$k$-ХАРАКТЕРЫ ФРОБЕНИУСА И $n$-КОЛЬЦЕВЫЕ ГОМОМОРФИЗМЫ
}

\author{
В. М. БУХШтАБЕР, Е. Г. РИС
}

Пусть $A$ и $B$ некоторые $\mathbf{Q}$-алгебры с единицей 1 , причем $B$ - коммутативная алгебра, где $\mathbf{Q}$ поле рациональных чисел. Для каждого линейного отображения $f: A \rightarrow B$ определим полилинейные отображения $\Phi_{k}(f): A^{k} \rightarrow B, k \geqslant 1$, при помощи рекуррентной формулы:

(1) $\Phi_{k+1}(f)\left(a_{1}, \ldots, a_{k+1}\right)=f\left(a_{1}\right) \Phi_{k}(f)\left(a_{2}, \ldots, a_{k+1}\right)$

$$
-\sum_{i=2}^{k+1} \Phi_{k}(f)\left(a_{2}, \ldots, a_{1} a_{i}, \ldots, a_{k+1}\right),
$$

где $\Phi_{1}(f)(a)=f(a)$.

ОПредЕЛЕниЕ 1 . Линейное отображение $f: A \rightarrow B$ называется фробениусовым $n$-гомоморфизмом (кратко, $\Phi$ - $n$-гомоморфизмом), если $\Phi_{n+1}(f) \equiv 0$.

В случае, когда $A$ - групповая алгебра $C(G)$ группы $G$ и $B=\mathbf{C}$ - поле комплексных чисел, формула (1) для $f$, определяемого характером $n$-мерного комплексного представления группы $G$ задает классический $(k+1)$-характер Фробениуса этого представления, причем $\Phi_{n+1}(f) \equiv 0$ (см. [1]).

Лемма 1. Пусть $f: A \rightarrow B$ - некоторый $\Phi$ - $n$-гомоморфизм, причем $B$ не имеет делителей нуля. Тогда, если $\Phi_{n}(f) \neq 0$, то $f(1)=n$. Следовательно, в общем случае $f(1) \in\{0,1, \ldots, n\}$.

Более трудным является следующий резултат.

ЛЕмма 2. В условиях леммы 1

$$
f\left(a_{1} a_{2}\right)=f\left(a_{2} a_{1}\right) \quad \text { для }
$$

Запишем перестановку $\sigma \in \Sigma_{k+1}$ в виде произведения попарно независимых циклов $\gamma_{1} \cdot \gamma_{2} \cdot \ldots \cdot \gamma_{r}$, включая и циклы длины 1. Для каждого цикла $\gamma=\left(i_{1}, \ldots, i_{q}\right)$ введем отображение $f_{\gamma}\left(a_{1}, \ldots, a_{k+1}\right)=f\left(a_{i_{1}} \cdot a_{i_{2}} \cdots a_{i_{q}}\right)$ и положим $f_{\sigma}=f_{\gamma_{1}} f_{\gamma_{2}} \cdots f_{\gamma_{r}}$.

ТЕОрема 1. Если $f$ является $\Phi$-n-гомоморфизмом для некоторого $n$ и $f\left(a_{1} a_{2}\right)=$ $f\left(a_{2} a_{1}\right)$ (см. лемму 2), mо

$$
\Phi_{k+1}(f)\left(a_{1}, \ldots, a_{k+1}\right)=\sum_{\sigma \in \Sigma_{k+1}} \operatorname{sign}(\sigma) f_{\sigma}\left(a_{1}, \ldots, a_{k+1}\right) .
$$

Для классических $k$-характеров Фробениуса формула (2) была получена в [2].

Наши исследования по теории многозначных групп (см. [3]-[5]) привели к следующему понятию.

ОПредЕлЕниЕ 2 (ср. с [5]). Линейное отображение $g: A \rightarrow B$ называется

- алгебраическим степени $n$ в $a \in A$, если существует полином

$$
p(a, t)=t^{n}-\beta_{1}(a) t^{n-1}+\cdots+(-1)^{n} \beta_{n}(a), \quad \beta_{q}(a) \in B
$$

такой что

$$
\sum_{q \geqslant 0} \frac{g\left(a^{q}\right)}{t^{q+1}}=\frac{1}{n} \frac{d}{d t} \ln p(a, t) ;
$$

- $n$-кольцевым гомоморфизмом, если оно алгебраическое степени $n$ для всех $a \in A$.

Непосредственно из определения 2 вытекает:

- если отображение является алгебраическим степени $n$ в $1 \in A$, то $g(1)=1$.

- если отображения $g_{1}$ и $g_{2}$ являются $n_{1}$ - и $n_{2}$-алгебраическими в $a \in A$, то отображение $g=\frac{1}{n_{1}+n_{2}}\left(n_{1} g_{1}+n_{2} g_{2}\right)$ является $\left(n_{1}+n_{2}\right)$-алгебраическим в $a \in A$. 
ЛЕмма 3. Если g является 1-кольиевым гомоморфизмом, то

$$
(g(a b)-g(a) g(b))^{2}=0 .
$$

Лемма 4. Следующие условия на линейнье отображения $g: A \rightarrow B$ являются әквивалентными:

1) $g$ - алгебраическое степени $n$ в $а \in A$;

2) $g(1)=1$ и для отображения $f=n g$ имеет место тождество

$$
\Phi_{n+1}(f)\left(a^{m}, a, \ldots, a\right) \equiv 0 \quad \text { для всех } \quad m \geqslant 1 .
$$

СледСтвиЕ 1. Если линейное отображение $f: A \rightarrow B$ является $\Phi$-n-гомоморфизмом u $f(1)=n$, то отображение $g=f / n$ является $n$-кольчевым гомоморфизмом.

Для каждого линейного отображения $g: A \rightarrow B$, такого что $g(1)=1$ введем отображения $\Psi_{k}(g): A^{k} \rightarrow B$ следующим образом: $\Psi_{1}(g)=g$ и $\Psi_{k+1}(g)\left(a_{1}, \ldots, a_{k+1}\right)=$ $\sum \sigma \in \Sigma_{k+1} \operatorname{det} M(\sigma)$, где $M(\sigma)=\left(m_{r, s}(\sigma)\right)$ и $m_{r, s}(\sigma)=0$ для всех $s>r+1, m_{r, r+1}(\sigma)=$ $r / n, m_{s+t, s}(\sigma)=g\left(a_{\sigma(s)} a_{\sigma(s+1)} \cdots a_{\sigma(s+t)}\right)$ для $t \geqslant 0$. Непосредственная проверка показывает, что $\Psi_{k}(g)$ являются полилинейньми отображениями для всех $k \geqslant 1$.

Теорема 2. Линейное отображение $g: A \rightarrow B$ c $g(1)=1$ является $n$-кольцевым тогда и только тогда, когда $\Psi_{n+1}(g) \equiv 0$.

Из теорем 1 и 2 вытекает важньй резултат.

СлЕДСтвиЕ 2. Если линейное отображение $g: A \rightarrow B$ является $n$-кольиевым и $g(a b)=g(b a)$ для всех $a, b \in A$, то отображение $f=n g$ является $\Phi$ - $n$-гомоморфизмом.

Множество всех $n$-кольцевых гомоморфизмов из $A$ в $B$ обозначим через $R^{(n)}(A, B)$. Аналогично классическому случаю $n=1$, задача вычисления $R^{(n)}(A, B)$ для $n>1$ лежит в основе теории двойственности $n$-алгебр Хопфа, введенных в [3], [5].

Пусть $(X)^{n}=X \times \cdots \times X / \Sigma_{n}-n$-я симметрическая степень множества $X$. Для $X=$ $R^{(1)}(A, B)$ определено вложение

$$
i_{x}:(X)^{n} \rightarrow R^{(n)}(A, B):\left[g_{1}, \ldots, g_{n}\right] \rightarrow \frac{1}{n} \sum g_{k} .
$$

Приведем первые следствия приведенных вьше результатов: вложение $i_{x}$ является взаимно однозначным для $B=\mathbf{C}$ и

1) $A=\mathbf{C}[t], X=\mathbf{C}$, т.е. $R^{(n)}(\mathbf{C}[t], \mathbf{C}) \cong \mathbf{C}^{n}$

2) $A=\mathbf{C}\left[t_{1}, \ldots, t_{m}\right], X=\mathbf{C}^{m}, n=2$, т.е. $R^{(2)}\left(\mathbf{C}\left[t_{1}, \ldots, t_{m}\right], \mathbf{C}\right) \cong\left(\mathbf{C}^{m}\right)^{2}$;

3) $A=\mathbf{C}^{m}$ - кольцо кольцо функций на конечном множестве $X=\left(x_{1}, \ldots, x_{m}\right)$, т.е. $R^{(n)}\left(\mathbf{C}^{m}, \mathbf{C}\right) \cong(X)^{n}$.

Мы благодарим John McКау, которьй обратил наше внимание на классические $k$-характеры Фробениуса при обсуждении резултатов [3] об $n$-колцевых гомоморфизмах.

\section{СПИСОК ЛИТЕРАТУРЫ}

[1] Frobenius G. Sitzungber // Preuss. Akad. Wiss. Berlin, 1896. P. 1343-1382. [2] Formanek E. The polynomial identities and invariants of $n \times n$ matrices // Regional Conferences. 1991. V. 78. [3] Buchstaber V., Rees E. Multivalued groups, their representations and Hopf algebras // Preprint MS-96-013: University of Edinburgh, 1996. [4] Buchstaber V., Rees E. Multivalued groups, $n$-Hopf algebras and $n$-ring homomorphisms // Preprint MS-96-017: University of Edinburgh, 1996. [5] Бухштабер В., Рис Е. // УМН. 1996. Т. 52. № 4. С. 149-150.

Московский государственньй

Принято редколлегией университет им. М. В. Ломоносова; 12.03.1997

University of Edinburgh;

J. C. Maxwell Building 